\title{
Constraints in the Evolution of Sex Ratio Adjustment
}

\author{
Stuart A. West ${ }^{1 *}$ and Ben C. Sheldon ${ }^{2}$
}

\begin{abstract}
When the relative fitness of male and female offspring varies with environmental conditions, evolutionary theory predicts that parents should adjust the sex of their offspring accordingly. Qualitative and even quantitative support for this prediction is striking in some taxa but much less convincing in others. Explaining such variation across taxa in the fit of sex ratio theory remains a major challenge. We use meta-analysis to test the role of two constraints in the evolution of sex ratios. Based on analysis of sex ratio skews in birds and wasps, we show that (i) mechanisms of sex determination do not necessarily constrain the evolution of sex ratio adjustment, and (ii) parental ability to predict their offsprings' environment influences the evolution of sex ratio patterns across taxa. More generally, our results show that multiple constraints may determine the precision of adaptation.
\end{abstract}

Trivers and Willard (1) were the first to suggest that parents should adjust the sex of their offspring in response to environmental conditions. They envisaged a mammal population in which (i) females in better condition have more resources for reproduction and so produce higher quality offspring, and (ii) competition for mates among males is intense, with only the highest quality males being successful, so that sons benefit more from increased resources than do daughters. In this case, they predicted that high-condition females should produce sons, and lowcondition females should produce daughters. Although the extent to which data on mammals fit this prediction is disputed, the same logic has been applied with considerable success to explain and predict the adjustment of offspring sex ratios (the proportion that is male) across a wide range of organisms in response to a multitude of environmental factors $(2-5)$.

In animals, the mechanism of sex determination is often suggested to be the major constraint determining the amount of adaptive sex ratio adjustment that is observed. Striking sex ratio adjustment is commonly seen in insects (ants, bees, and wasps) in which haplodiploid sex determination may give females precise control over the sex of their offspring according to whether they fertilize eggs (males develop from unfertilized eggs, and females develop from fertilized eggs) $(2,4)$. Sex ratio adjustment is much less often seen in vertebrates with chromosomal (genetic) sex determination (CSD), perhaps because random meiosis would lead

${ }^{1}$ Institute of Cell, Animal, and Population Biology, University of Edinburgh, Edinburgh EH9 3JT, UK. '2Edward Grey Institute, Department of Zoology, University of Oxford, Oxford, UK.

*To whom correspondence should be addressed. Email: stu.west@ed.ac.uk to a mean sex ratio of 0.5 , with binomial variance around this mean, reducing the scope for sex ratio adjustment because of the need to sacrifice eggs or embryos $(2,6-10)$.

Alternative explanations have been proposed to account for less striking sex ratio adjustment in vertebrates, such as multiple factors influencing sex ratio evolution, more complex life histories, or variation in the reliability with which offspring fitness can be predicted (environmental predictability) (5, 9-11). In addition, recent studies of mammals, birds, and other taxa with CSD (frogs, lizards, snakes, and spiders) have reported striking shifts in offspring sex ratios in manners consistent with adaptation $(5,12-18)$. Although these data suggest that sex ratios might not be completely constrained by CSD, other authors have suggested that there is no consistent pattern and that statistically significant cases may represent sampling error or publication bias (a tendency to publish only those studies reporting a positive or significant effect) $(7,19-23)$.

Resolution of these differing points of view requires comparisons across taxa. Here, our aims are to (i) determine whether CSD prevents facultative sex ratio adjustment, and (ii) test the alternative prediction that the extent of sex ratio adjustment can be influenced by environmental predictability.

Do vertebrates with CSD show consistent sex ratio patterns? Previous studies examining broad-scale patterns of sex ratio variation in vertebrates have tended to focus on population sex ratios or sex ratio variance $(7,19,22,24)$. However, if individuals are selected to adjust their offspring sex ratio facultatively, with some individuals producing sons and others daughters, then the overall population sex ratio is very difficult to predict $(2,3,10,25-27)$. Consequently, examining population sex ratios is a poor test of individual adaptation, and it is not surprising that no consistent patterns have been found. The solution to this problem is, rather than considering population sex ratios, to examine the precision with which individuals facultatively adjust offspring sex ratios. We do this by using meta-analytic techniques, in which a standard measure of statistical effect size from each study (the correlation coefficient $r$ ) is used as the response variable in comparative analyses $(28,29)$. By carrying out analyses with effect size obtained from different studies as the response variable, we can test whether sex ratios are consistently adjusted in the direction predicted and compare the relative precision of sex ratio manipulation across groups or taxa.

A second problem is that not all studies are equally suitable for an analysis of this kind. For most studies on sex ratio variation in birds and mammals, the expected sex ratio shift depends on life history details that are not known and thus cannot be predicted a priori (30). We avoid this problem by restricting our meta-analysis to cases in which there is a clear theoretical prediction as to the direction of an effect. In birds and mammals, this reduces to only two situations. First, females are predicted to adjust their sex ratio in response to mate attractiveness, producing more sons when mated to an attractive male (31-33). This is predicted because males show greater variation in reproductive success than females (34), and so sons stand to benefit more from having a high-quality father. Second, in cooperatively breeding species where one sex helps (in the rearing of subsequent offspring) more than the other, it is predicted that when an individual (or group) lacks helping individuals, they should bias their offspring sex ratio toward the sex that provides greater levels of help $(13,26$, $35)$.

We located published data from 11 studies of eight bird species testing the hypothesis that sex ratio should be adjusted to mate attractiveness, and we also found data from five studies of four bird species testing the hypothesis that sex ratio should be adjusted in response to helper status in cooperatively breeding species (36). In all cases, we assigned a positive value to the effect size $(r)$ if the sex ratio shift was in the predicted direction and a negative value if it was in the opposite direction. We used standard metaanalytic procedures and relatively conservative (mixed model) statistical tests (37).

Overall, these studies show consistent facultative adjustment of offspring sex ratios by birds in the direction predicted by theory, with the average standardized effect size $(r)$ being significantly greater than zero (Table 1) (Fig. 1). This result holds when using each study as an independent data point, when averaging to obtain only one data point from each species, when averaging to obtain only 
RESEARCH ARTICLE

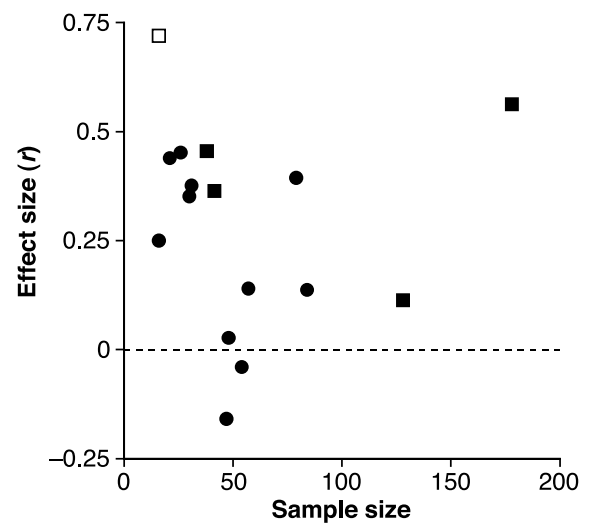

Fig. 1. Variation in facultative sex ratio adjustment by birds and a mammal. The effect size $(r)$ is plotted against the sample size of the study. A positive value of $r$ corresponds to a sex ratio shift in the predicted direction, and a negative value corresponds to a shift in the opposite direction. The significant tendency toward positive values indicates a consistent trend to adjust sex ratios as predicted by theory. The different symbols represent sex ratio adjustment by birds in response to mate quality (solid circles) or number of helpers (solid squares), and by a mammal in response to the number of helpers (open square) (36).

one data point from each family (to control for possible phylogenetic artifacts), or when considering each hypothesis (mate attractiveness and helper status) separately (Table 1). In addition, several analyses suggest that publication bias $(22,29)$ does not greatly affect the overall pattern identified here (38). We are aware of only one study of these patterns in a mammal: a study of sex ratios in relation to the presence of helpers in African wild dogs $(36,39)$. Adding this study gives an overall weighted mean effect size of $r=$ 0.289 at the level of studies [95\% confidence interval (CI): 0.175 to $0.419 ; n=16$ studies].

Overall, these results suggest that 3 to $18 \%$ of the variance in sex ratio among families can be explained by facultative sex ratio adjustment where these two hypotheses apply. These effects are quite small, and power analysis shows that the sample size required to reject the null hypothesis with $80 \%$ certainty at $P=0.05$, with $r=0.252$ (the mean value for bird species) is 120 . A sample size this large is rarely used in field studies of behavioral ecology [for example, in only 2 out of 17 of the studies on which our analyses are based (Fig. 1) (36)], suggesting that the likelihood of type II error (failing to detect real effects) may be high in this field.

Environmental predictability and precision of sex ratio adaptation. The analyses above suggest that CSD does not necessarily prevent facultative sex ratio adjustment. Given this, we now focus on an alternative factor that has been suggested to explain variation in the extent of sex ratio adjustment: environmental predictability

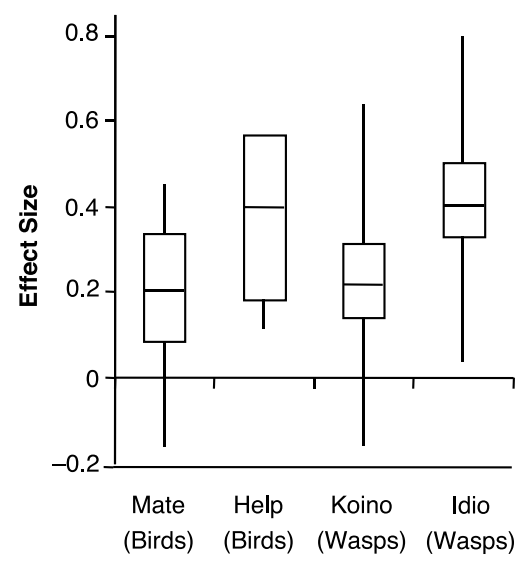

Fig. 2. Sex ratio adjustment across taxa. Plotted are the mean (horizontal line), $95 \% \mathrm{Cl}$ (box), and range (vertical line) of the effect size $(r)$ for different groups. A positive value of $r$ corresponds to a sex ratio shift in the predicted direction and a negative value corresponds to a shift in the opposite direction. The different columns show the effect size for variation in offspring sex ratio for birds in response to mate quality, for birds in response to helper status, and for parasitic wasps that do (idiobionts) and do not (koinobionts) kill the host when an egg is laid in response to host size.

$(2,5,9-11)$. If environmental factors that cause differences in the reproductive value of males and females are difficult to predict, then strongly skewed sex ratios may in some cases lead to the overproduction of the sex with the lower reproductive value. Consequently, lower environmental predictability will lead to weaker selection for sex ratio adjustment.

We compared the extent of sex ratio adjustment shown in birds with that in parasitic wasps (parasitoids). Parasitic wasps are insects whose larvae develop by feeding on the bodies of other arthropods. We compared data from parasitic wasps with data from birds because their haplodiploid sex determination should leave sex ratios unconstrained. They also offer an excellent opportunity for testing the importance of environmental predictability, because numerous data are available for a comparison in which the predictability of the environment will vary $(36,40)$. In many species in which only one individual can develop per host (termed solitary), females lay male eggs on small hosts and female eggs on large hosts. This is presumed to be advantageous because females gain a greater benefit from the resulting increase in body size than do males $(4,41)$. In some species (idiobionts), females kill or paralyze the host at oviposition, and in such cases host size will be a reliable cue as to the resources that offspring will have available for development. However, in other species (koinobionts), the host is not killed by the female at oviposition, and so continues to grow, in which case host size at oviposition is a less

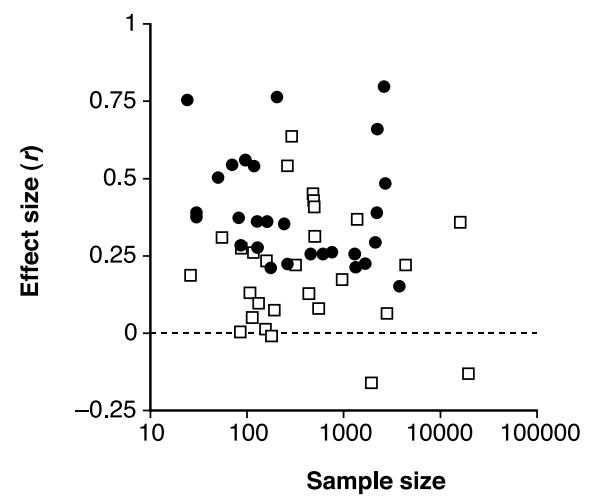

Fig. 3. Facultative sex ratio adjustment by parasitoid wasps in relation to life history. The effect size $(r)$ is plotted against the sample size of the study (note log scale). A positive value of $r$ corresponds to a sex ratio shift in the predicted direction (more females on larger hosts), and a negative value corresponds to the opposite direction. The different symbols represent species in which the host is (idiobionts, solid circles) and is not (koinobionts, open squares) killed when an egg is laid. Data were obtained from 65 studies on 56 parasitic wasp species (28 koinobiont and 28 idiobiont species) (36). Idiobionts show significantly higher mean effect sizes [studies as independent data points, $Q_{B}=6.08, P=0.005$; species as independent data points, $Q_{B}=8.21, P=0.002$; formal comparative analysis by the method of independent contrasts, $n=6$ independent contrasts, mean contrast in effect size $=-0.092$ (95\% Cl: $-0.014,-0.174 ; P=0.03)]$.

reliable predictor of the resources that offspring will have available for development. This leads to the prediction that because environmental predictability is lower for koinobionts, they should exhibit less extreme facultative sex ratio variation (40).

Comparison of effect size $(r)$ among the different groups of wasps (koinobiont and idiobiont) and birds (helper status or mate quality) revealed significant heterogeneity in effect size (Fig. 2) [between-group heterogeneity $\left(Q_{\mathrm{B}}\right)=10.01$, df $=3, P=0.009$; using species mean values]. This heterogeneity was caused by the effect size in idiobiont wasps and birds in response to helper status being significantly higher than that in koinobiont wasps and birds in response to mate quality $\left(Q_{\mathrm{B}}=9.93, \mathrm{df}=1, P=0.001\right)$. Within these two groups, there were no significant differences when the following comparisons were made: idiobiont wasps versus birds in response to helper status $\left(Q_{\mathrm{B}}=0.057, \mathrm{df}=1\right.$, $P=0.78$ ) and koinobiont wasps versus birds in response to mate quality $\left(Q_{\mathrm{B}}=0.084\right.$, $\mathrm{df}=1, P=0.75$ ).

These results provide strong support for the importance of environmental predictability. As predicted, within parasitic wasps, we found that species in which the host was killed when an egg was laid (idiobionts) showed significantly greater sex ratio skews than did species in which the host was not 
RESEARCH ARTICLE

Table 1. Summary of standardized effect sizes for studies of individual sex ratio adjustment in various groups of birds and parasitic wasps.

\begin{tabular}{lllrr}
\hline \multicolumn{1}{c}{ Class of study } & Mean $r$ & $95 \% \mathrm{Cl}$ & $n$ studies & $X \dagger$ \\
\hline All birds, studies independent & $0.252^{* *}$ & $0.132-0.384$ & 15 & 57 \\
All birds, species independent & $0.261^{* *}$ & $0.121-0.391$ & 12 & 39 \\
All birds, families independent & $0.252^{* *}$ & $0.150-0.369$ & 9 & -14 \\
All birds, studies independent & $0.220^{* *}$ & $0.116-0.341$ & 11 & 74 \\
All birds, species independent+ & $0.217^{* *}$ & $0.090-0.350$ & 11 & 37 \\
Mate, studies independent & $0.205^{*}$ & $0.088-0.335$ & 8 & 45 \\
Mate, species independent & $0.187^{*}$ & $0.041-0.340$ & 4 & 8 \\
Help, species independent & $0.397^{*}$ & $0.191-0.566$ & 65 & 8 \\
All wasps, studies independent & $0.319^{* *}$ & $0.258-0.383$ & 56 & $\mathbf{1 4 5 1}$ \\
All wasps, species independent & $0.328^{* *}$ & $0.262-0.396$ & 36 & $\mathbf{1 2 0 6}$ \\
Idiobionts, studies independent & $0.405^{* *}$ & $0.318-0.494$ & $\mathbf{5 9 9}$ \\
Idiobionts, species independent & $0.440^{* *}$ & $0.353-0.544$ & $\mathbf{2 8}$ & $\mathbf{5 1 5}$ \\
Koinobionts, studies independent & $0.217^{* *}$ & $0.140-0.296$ & $\mathbf{2 9}$ & $\mathbf{1 8 2}$ \\
Koinobionts, species independent & $0.219^{* *}$ & $0.137-0.305$ & 28 & $\mathbf{1 7 3}$ \\
\hline
\end{tabular}

$* p<0.01{ }^{* *} p<0.001 . \quad \dagger X$ is the fail-safe number, which is the number of unpublished studies averaging zero effect size that would have to exist for the overall mean effect size to be not significantly different from zero, calculated following (29); values in bold are robust, under the criterion that a fail-safe number is robust when $X>5 n+$ 10. $\quad$ Excluding the Seychelles warbler

killed (koinobionts) (Table 1) (Fig. 3). Approximately four times as much variance in the offspring sex ratio is explained by host size in idiobionts $(19 \%$ at the level of the species) as in koinobionts (5\%). Among birds, the relevant environmental variables may be easier to assess in relation to helper status (number of helping offspring and territory quality) than in relation to mate quality. This may explain why the amount of sex ratio adjustment is greater in response to helper status $(16 \%$ of the variance) than to mate quality (4\%), although this difference is not significant if comparison is made on only the bird data $\left(Q_{\mathrm{B}}=1.27, P=0.26\right)$.

Despite the different methodologies used, these data suggest that sex ratio shifts are not necessarily more extreme in haplodiploid species such as wasps than in species with CSD, such as birds. Hence, the mechanism of sex determination, and any constraint that this may place on sex ratio adaptation, is not sufficient to explain all variation in adaptation in sex ratio. Instead, it is necessary to consider and compare different categories within various taxa (such as idiobiont versus koiniobiont, helper status, or mate quality). Explaining how facultative sex ratio adjustment is achieved with CSD remains a major theoretical and empirical challenge (21).

Precision of adaptation and a costbenefit approach. A single unifying framework, rather than concentration on specific potential constraints to adaptation, is required to explain variation in the extent to which different species adjust their offspring sex ratio. We suggest the use of a cost-benefit approach. Specifically, that facultative sex ratio variation will only be favored when the fitness benefits of this behavior are greater than its costs. In cases where facultative sex ratio variation is favored, it will evolve to a level where the benefits of any further (mar- ginal) increase in the precision of sex ratio adjustment would be balanced by its cost. Consequently, the most extreme and precise sex ratio variation will be seen in species in which the fitness benefits of facultative sex ratio adjustment are high and the costs low. This approach allows for the possibility that the mechanism of sex determination will be important [for example, facultative sex ratio variation may be more costly with CSD (2, $8-10,42)$ ] but that it is only one of a number of factors that must be taken into account. The benefit of facultative sex ratio variation will depend heavily on how much fitness gain is to be made from shifting offspring sex ratios, which will be influenced by the strength and form of selection involved (43, 44 ), and hence factors such as environmental predictability (45). More generally, understanding the relative importance of different potential constraints on adaptation remains one of the biggest problems for evolutionary biology (44). The extremely close fit that can be expected between the predictions of relatively simple theoretical models and empirical data means that the study of sex ratio patterns provides an excellent model for addressing this problem.

\section{References and Notes}

1. R. L. Trivers, D. E. Willard, Science 179, 90 (1973).

2. E. L. Charnov, The Theory of Sex Allocation (Princeton Univ. Press, Princeton, NJ, 1982).

3. S. A. Frank, Annu. Rev. Ecol. Syst. 21, 13 (1990).

4. H. C. J. Godfray, Parasitoids: Behavioural and Evolutionary Ecology (Princeton Univ. Press, Princeton, NJ, 1994).

5. B. C. Sheldon, Heredity 80, 397 (1998).

6. J. Maynard Smith, The Evolution of Sex (Cambridge Univ. Press, Cambridge, 1978).

7. G. C. Williams, Proc. R. Soc. London Ser. B 205, 567 (1979).

8. J. Maynard Smith, Behav. Ecol. Sociobiol. 7, 247 (1980).

9. O. Leimar, Behav. Ecol. 7, 316 (1996).

10. I. Pen, F. J. Weissing, in Sex Ratios: Concepts and
Research Methods, I. C. W. Hardy, Ed. (Cambridge Univ. Press, Cambridge, 2002) pp. 26-45.

11. S. A. West, E. A. Herre, B. C. Sheldon, Science 290, 288 (2000).

12. T. Madsen, R. Shine, Evolution 46, 1549 (1992).

13. J. Komdeur, S. Daan, J. Tinbergen, C. Mateman, Nature 385, 522 (1997).

14. L. Aviles, J. McCormack, A. Cutter, T. Bukowski, Proc. R. Soc. London Ser. B 267, 1445 (2000).

15. Y. Sakisaka, T. Yahara, I. Miura, E. Kasuya, Mol. Ecol. 9, 1711 (2000).

16. C. N. Johnson et al., Proc. R. Soc. London Ser. B 268, 2001 (2001).

17. M. Olsson, R. Shine, J. Evol. Biol. 14, 120 (2001)

18. A. V. Badyaev et al., Science, 295, 316 (2002).

19. T. H. Clutton-Brock, Ibis 128, 329 (1986).

20. J. J. Bull, E. L. Charnov, in Oxford Surveys in Evolutionary Biology, P. H. Harvey, L. Partridge, Eds. (Oxford Univ. Press, Oxford, 1988), vol. 5, pp. 96-135.

21. S. Krackow, Proc. 22 Int. Ornithol. Congr. 425 (1999).

22. A. R. Palmer, Annu. Rev. Ecol. Syst. 31, 441 (2000).

23. G. R. Brown, Anim. Behav. 61, 683 (2001).

24. T. H. Clutton-Brock, G. R. Iason, Q. Rev. Biol. 61, 339 (1986).

25. S. A. Frank, Theor. Popul. Biol. 31, 47 (1987).

26. I. Pen, F. J. Weissing, Proc. R. Soc. London Ser. B 267 539 (2000).

27. The population sex ratio depends on a range of factors that are rarely known, such as the details of male and female life histories, distribution of variation in maternal quality, the extent to which other behaviors (such as clutch size) are facultatively adjusted, and the form of the trade-off between current and future reproduction $(3,10,25,26,36)$.

28. The correlation coefficient obtained from a particular study provides an estimate of how precisely individuals adjust their offspring sex ratios. $r$ ranges between \pm 1 , with values close to 1 (or to -1 ) implying an extremely precise (low scatter) positive (or negative) shift in the offspring sex ratio in response to variation in the relevant environmental variable; a value of 0 represents no correlation. More specifically, $r^{2}$ from a particular study represents the proportion of variance in the offspring sex ratio that is explained by the explanatory variable.

29. R. Rosenthal, Meta-Analytic Procedures for Social Research (Sage Publications, Thousand Oaks, CA, 1991)

30. For example, consider Trivers and Willard's (1) prediction for mammals given in the main text. Theoretical modeling has shown that if there is strong mother-daughter transmission of condition, such as through inheritance of territories, then the optimum strategy may be for females in good condition to produce daughters (9). Consequently, there is no clear prediction against which to test studies relating maternal condition to offspring sex ratio, in the absence of detailed case-by-case studies of the influence of maternal condition on the reproductive value of sons and daughters. Similarly, many studies of bird sex ratios report associations with variables such as maternal age, condition, or time within the breeding season (5). Although post-hoc explanations of these patterns can be offered, there is usually little evidence to support these explanations [for an exception see (18)]. Because for most of these studies we know neither what direction any effect should lie in, nor even whether there should be an effect, it is not possible to use them to assess whether a general pattern of sex ratio variation occurs across studies.

31. N. Burley, Science 211, 721 (1981)

32. Evolution 40, 1191 (1986).

33. I. Pen, F. J. Weissing, Selection 1, 59 (2000).

34. A. P. Møller, P. Ninni, Behav. Ecol. Sociobiol. 43, 345 (1998).

35. P. A. Gowaty, M. R. Lennartz, Am. Nat. 126, 347 (1985).

36. Supplemental material is available on Science Online at www.sciencemag.org/cgi/content/full/1069043/ DC1.

37. We followed standard meta-analysis practices, described in detail elsewhere $(29,36,46)$. We used mixed model analysis for the meta-analysis, because our underlying hypothesis was that effect sizes would vary among species. Mixed models are appropriate for meta-analyses of ecological data, in that mixed 
RESEARCH ARTICLE

models are more robust than fixed-effect models because they allow simultaneously for variation in effect sizes due to within-study sampling error and between-study variation in effect size (46). Repeating analyses using fixed-effect models yielded similar conclusions, except that differences among categories were more likely to be judged significant and fail-safe numbers were much larger.

38. Several lines of evidence suggest that publication bias does not greatly affect the overall pattern identified here. First, scatter plots of effect size versus sample size (Figs. 1 and 3) do not show the pattern suggestive of selective reporting: a funnel with missing values for effect sizes close to zero with small sample sizes (22). Second, the rank correlation between effect size and sample size $\left(r_{\text {bias }}\right)$ for the bird studies was not significantly negative [Spearman rank correlation coefficient $\left(r_{\mathrm{s}}\right)=-0.26, n=15$ studies, $P=0.32$; when the Seychelles warbler was excluded, $r_{\mathrm{s}}=-0.39, n=$ 14 studies, $P=0.17$ ], which would provide evidence for publication bias (22). Third, very conservative tests were significant. Taking all bird studies, 13 out of 15 estimates (sign test: $P=0.007$ two-tailed), 10 out of 12 species means (sign test: $P=0.039$, two-tailed), and 8 out of 9 family means (sign test: $P=0.039$, two-tailed) showed positive effect sizes. Fourth, although transformed effect sizes for all studies did not show significant heterogeneity among studies $\left(\chi_{(14)}^{2}=10.18, P=\right.$ 0.75 ), we also examined the data excluding the study of the Seychelles warblers (13), which had the largest effect size, and the largest sample size. Weighted mean effect size was still significantly greater than zero, when studies or species were considered to be independent (Table 1). Fifth, we calculated the number of unpublished studies averaging zero effect size that would have to exist for the overall mean effect size to be not significantly different from zero (Table 1). Interpretation of the meaning of a "fail-safe number" depends in part on subjective assessment of whether it is likely that so many unpublished studies exist (29); we consider it unlikely. A quantitative criterion is that a result should be regarded as robust if the fail-safe number, $X$, exceeds $5 n+10$, where $n$ is the number of studies on which the meta-analyses were based (29). By this criterion, none of these results is robust (Table 1 ), but the sample size is rather small.

39. S. Creel, N. M. Creel, S. L. Monfort, Anim. Reprod. Sci. 53, 315 (1998)

40. B. H. King, Oecologia 78, 420 (1989).

41. E. L. Charnov, R. L. Los-den Hartogh, W. T. Jones, J. van den Assem, Nature 289, 27 (1981).

42. I. Pen, F. J. Weissing, S. Daan, Am. Nat. 153, 384 (1999).

43. Nature 329, 627 (1987)

44. E. A. Herre, C. A. Machado, S. A. West, in Adaptionism and Optimality, S. Orzack, E. Sober, Eds. (Cambridge Univ. Press, Cambridge, 2001), pp. 191-218.

45. Consistent with the predicted importance of the strength and form of selection; (i) fig wasp species show more extreme sex ratio shifts in more variable environments, where there is stronger selection to adjust sex ratios in response to environmental conditions $(43,44)$, and more precise (lower variance) sex ratios in situations where selection for precise sex ratios (stabilizing selection) is greater (47); (ii) in species where the method of sex determination may impose a heavy constraint (such as CSD and pseudo-arrhenotoky in spiders, aphids, snakes, and mites), many cases of extreme (and precise) sex ratio variation occur when there is intense competition among brothers for mates (local mate competition) and strong selection for extremely female-biased sex ratios (such as $5 \%$ males) $(12,14,48,49)$; and (iii) environmental predictability could be important in explaining broad taxonomic patterns, because in many cases it would seem likely to be easier for insects such as parasitic wasps to assess relevant factors than for a vertebrate to do so (11).

46. J. Gurevitch, L. V. Hedges, Ecology 80, 1142 (1999).

47. S. A. West, E. A. Herre, Evolution 52, 475 (1998).

48. Y. Yamaguchi, Nature 318, 460 (1985).

49. C. J. Nagelkerke, M. W. Sabelis, J. Evol. Biol. 11, 649 (1998).

50. We thank D. Allsop, F. Balloux, N. Colegrave, A Griffin, K. Lessells, I. Pen, S. Reece, L. Rowe, D. Shuker, and A. Sugden for useful discussion; and J. van Alphen, R. Belshaw, G. Broad, C. Godfray, J. Noyes, A. Rivero, and especially D. Quicke for supplying biological and taxonomic help. Funded by the UK Biotechnology and Biological Sciences Research Council, the National Environment Research Council (UK), and the Royal Society.

17 December 2001; accepted 24 January 2002 Published online 31 January 2002; $10.1126 /$ science. 1069043

Include this information when citing this paper.

\section{Extraction of Black Hole Rotational Energy by a Magnetic Field and the Formation of Relativistic Jets}

\author{
Shinji Koide, ${ }^{1}$ Kazunari Shibata, ${ }^{2}$ Takahiro Kudoh, ${ }^{3}$ \\ David L. Meier ${ }^{4}$
}

\begin{abstract}
Using numerical simulations, we modeled the general relativistic magnetohydrodynamic behavior of a plasma flowing into a rapidly rotating black hole in a large-scale magnetic field. The results show that a torsional Alfvén wave is generated by the rotational dragging of space near the black hole. The wave transports energy along the magnetic field lines outward, causing the total energy of the plasma near the hole to decrease to negative values. When this negative energy plasma enters the horizon, the rotational energy of the black hole decreases. Through this process, the energy of the spinning black hole is extracted magnetically
\end{abstract}

Relativistic jets have now been discovered in several different classes of astrophysical objects, including active galactic nuclei $(1,2)$, microquasars $(3,4)$, and gamma ray bursts

${ }^{1}$ Faculty of Engineering, Toyama University, 3190 Gofuku, Toyama 930-8555, Japan. ${ }^{2}$ Kwasan and Hida Observatory, Kyoto University, Yamashina, Kyoto, 607-8471, Japan. ${ }^{3}$ National Astronomical Observatory, Mitaka, Tokyo 181-8588, Japan. 4Jet Propulsion Laboratory, California Institute of Technology, Pasadena, CA 91109, USA.
(5). A rapidly spinning black hole may exist at the center of each of these objects, and energetic reactions that occur near the hole may be responsible for the jets. One of the most promising processes for producing relativistic jets is the extraction of rotational energy from a spinning (Kerr) black hole (6, 7). One method of extraction is the Penrose process, which uses fission of a particle near the black hole to extract the black hole rotational energy (6). However, this process may not be applicable to most astrophysical objects, because the particle fission must occur near the black hole, and the relative velocity of the particles produced by the fission should be near the speed of light. On the other hand, Blandford and Znajek (7) showed that a large-scale magnetic field around a Kerr black hole also could extract rotational energy. They assumed a magnetic force-free condition, which corresponds to an extremely strong magnetic field or an extremely low inertia plasma case. Recently, evidence of the extraction of rotational energy from a Kerr black hole by a magnetic field was suggested by observations of a broad $\mathrm{Fe} \mathrm{K} \alpha$ line in the bright Seyfert 1 galaxy MCG-6-30-15 (8). Modeling of this emission indicates that it is concentrated in a small central disk region near the black hole. It is plausibly explained by a model in which the black hole rotational energy is being extracted into the disk by a magnetic field with a strength of $\sim 10^{4}$ Gauss that connects the black hole to the disk.

To understand the basic physics of rotational energy extraction from a black hole with a finite magnetic field, we have investigated a somewhat simpler system using general relativistic magnetohydrodynamic (MHD) numerical calculations. Initially, the system consists of a Kerr black hole with a uniform magnetic field, uniform plasma, and no accretion disk. The calculations are based on the general relativistic formulation of the laws of conservation of particle number and 